\title{
¿KUHN FILÓSOFO DE LA CIENCIA?
}

Eloy RADA

Nunca he podido leer a Kuhn sin sufrir, a la vez, estímulos y sugerencias de la más variada índole y por otra parte sin dejar de experimentar un desasosiego particular, como si algo no identificado anduviese suelto por el desván de sus escritos. En las líneas que siguen trataré de acercarme a eso que, quizá, no sea más que el eco de mis propios fantasmas.

Desde la publicación de La Estructura de las Revoluciones Científicas, y sin el menor género de duda por su influencia, se han originado distintos debates, unos en torno a alguno de los conceptos más significativos utilizados por Kuhn, -nadie habrá olvidado las discusiones sobre los paradigmas, p.e.- otros en torno a 'posiciones' supuestamente kuhnianas, como el relativismo, el historicismo, ciertas formas de psicologismo o de sociologismo, etc. y alguno, más global, sobre el alcance mismo de la obra kuhnia$\mathrm{na}^{1}$. Él mismo gastó mucho tiempo y muchas energías en aclarar conceptos y giros metafóricos que, según decía, no habían quedado suficientemente claros, no se habían entendido bien o habían sido maliciosamente interpretados.

1 Por citar dos muy recientes: Paul Horwich (ed): World Changes. Thomas Kuhn and the Nature of Science. MIT Press. Cambridge. Massachusetts. 1993; y Paul Hoyningen-Huene: Die Wissenschaftsphilosophie Thomas S. Kuhns: Rekonstruktion und Grundlagenprobleme. Friedr. Vieweg \& Sohn Verlagsgesellschaft mbH, Braunschweig. 1989. trad. inglesa de Alexander T. Levine: Reconstructing Scientific Revolutions. Thomas S Kuhn's Philosophy of Science. The Univ. of Chicago Press. 1993.

Endoxa: Series Filosoficas, $n^{\circ}$ 9, 1997, UNED, Madrid:

Eloy Rada Garcia: ¿Kuhn filosofo de la ciencia?

pp. 77-100. 
Sin alejarse de esta perspectiva general cabe también la discusión sobre la naturaleza misma de esa obra -si filosofía de la ciencia o más bien historia de la ciencia- y, seguidamente, si ofició Kuhn de filósofo más que de historiador o al contrario, o quizá si se apoyó en la Historia de la Ciencia para hacer una 'filosofía ilustrada mediante ejemplos'.

Retrospectivamente podríamos situar a Kuhn en una línea de investigación de contextura histórica que, al menos, pasaría por W. Whewell ${ }^{3}$ y E. Mach $^{4}$ en el siglo XIX, continuada en este siglo por P. Duhem, ${ }^{5}$ E. Meyerson, ${ }^{6}$ E. Cassirer, ${ }^{7}$ E.A. Burtt, ${ }^{8}$ N. Hanson, ${ }^{9}$ S. Toulmin ${ }^{10}$ y sobre todos A. Koyré ${ }^{11}$ anteriores todos a la intervención del propio Kuhn con la publicación de su La Estructura de las Revoluciones científica en 1962. De esta somera relación de autores y obras también parece evidente, retrospectivamente hablando, que las Revoluciones de Kuhn causó un impacto mucho mayor que ningún otro, anterior o posterior.

2 "La enseñanza de la filosofía por medio del ejemplo" es la frase de Kuhn en "La función de la medición en la física moderna". La Tensión Esencial. MadridMéxico 1982, p. 203.

3 The Philosophy of the Inductive Sciences. Founded Upon Their History. 3 vols. J.W. Parker. Londres, 1847.

4 Die Geschichte und die Wurzel des Satzes von der Erhaltung der Arbeit. J.G. Clave'sche. Praga, 1872. Y también: Die Mechanik in ihrer Entwickelung. Historischkritisch dargestellt. L.A. Brokhaus. Leipzig, 1883. (Hay traducción al español: El desarrollo histórico-crítico de la mecánica. Espasa-Calpe. Madrid, 1955).

${ }^{5}$ La théorie physique: son objet et sa structure. Chevalier et Rivière. Paris, 1906.

6 Identité et realité. F. Alcan. París, 1908. (En español: Identidad y Realidad. Madrid: Reus, 1929.)

7 Das Erkenntnisproblem. 3 vols. B. Cassirer. Berlín, 1906-20.

8 The Metaphysical Foundations of Modern Science. Nueva York, 1924. Revisada en Routledge and Kegan Paul. Londres, 1949.

9 Patterns of Discovery. Cambridge University Press. Cambridge, 1958.

${ }^{10}$ Foresight and Understanding. Londres: Hutchinson y Harper and Row, Nueva York: Torchbook, 1963.

11 Por citar alguno de los más importantes a este propósito: Études galiléennes. Hermann, Paris, 1939. From the Closed World to the Infinite Universe. The Johns Hopkins Press, 1957. La révolution astronomique: Copernic, Kepler, Borelli. Hermann, París, 1961. 
Es un lugar común que 'más vale llegar a tiempo que rondar un año' y Kuhn cumplió doblemente con el recio aforismo. Estaba en el ambiente y ya habían aparecido algunas señales de esos aires nuevos insinuando que la estrecha racionalidad reconstructivista de la epistemología del Círculo de Viena, al igual que el racionalismo crítico de índole popperiana, daban poca cabida al problema más arduo que planteaba la ciencia a estas alturas de su historia: su desarrollo o, si se quiere, su dinámica histórica. Esto estaba debatiéndose así en la tradición anglosajona (Hanson, Toulmin, Feyerabend) que trataba de dar cuenta filosófica de la incongruencia entre la idea "acumulativa" más extendida y el "discontinuísmo" inherente al modelo falsacionista popperiano. $Y$ en esto apareció Kuhn con sus Revoluciones. Quizá la casualidad hizo que I.Lakatos anduviese entonces por algunas universidades de EE.UU. y se diera cuenta de la oportunidad que se presentaba para provocar la discusión filosófica $y$, de regreso a Inglaterra, impulsa la convocatoria de un Coloquio Internacional de Filosofía de la Ciencia, que tuvo lugar en el Bedford College de Londres en julio de 1965. El Coloquio puso frente a frente (¿se trataba de eso? ${ }^{12}$ ) a Kuhn y a Popper, dando a Kuhn la oportunidad de "desafiar" de igual a igual al más reconocido filósofo de la ciencia del momento. ${ }^{13}$

Se ha sugerido que este encuentro significó una contraposición, para algunos irreductible, entre Historia y Filosofía de la ciencia. ${ }^{14}$

12 Recordemos aquí que la intervención de Kuhn titulada "¿Lógica del descubrimiento o psicología de la investigación?" se inicia con las siguientes palabras: "Me propongo oponer en estas páginas el punto de vista del desarrollo científico, cuyas lineas generales se encuentran en mi libro La Estructura de las Revoluciones Científicas, a los puntos de vista de nuestro presidente, Sir Karl Popper, que son mejor conocidos".

${ }_{13}$ "There is no philosopher writing in English who can match Karl Popper in range or in the quality of his work... (Bryan Magee). Así reza la contraportada de Unended Quest, la Autobiografía, en la edición de Fontana-Collins. Londres, 1976.

14 Los hitos más significativos, aunque no los únicos, y por su orden de publicación, serían los siguientes:

1970.- Stuewer, Roger H.: Historical and Philosophical Perspetives of Science (ed.). University of Minnesota Press, Minneapolis. (Minnesota Studies in the Philosophy 
De hecho significó, al menos, la puesta en tela de juicio de un modo de hacer filosofía de la ciencia heredado del viejo positivismo del s.XIX, tal y como había sido canonizado por los miembros del Círculo de Viena y sus afines por una parte, y por Popper y los suyos por otra, pese a los muchos puntos de coincidencia que Kuhn confiesa con los de Sir Karl Popper.$^{15}$ El desarrollo científico concebido por los positivistas como acumulación de conocimientos resultó directamente afectado por el análisis de Kuhn y, como consecuencia, etiquetado de "insuficiente". De manera menos directa -aunque no menos contundente- entraba en crisis el racionalismo crítico popperiano, en la confrontación directa entre falsacionismo y revolución científica. No me parece posible aquí, ni resumir las ya muy largas controversias y evaluaciones habidas hasta la fecha, ni siquiera decantarme por alguna de las posturas adoptadas en alguna de las más célebres. Simplemente haré algunas observaciones, cualquiera que sea el lado del que vayan a caer. La pregunta subyacente a todas ellas sería: ¿El artefacto

of Science. vol. V.)

1973.- Giere, Ronald: "History and Philosophy of Science: Intimate Relationship or Marriage of Convenience?" en British Journal for the Philosophy of Science, 24, pp.282-297.

1974.- Musgrave, Alan: "Logical versus Historical Theories of Confirmation", en British Journal for the Philosophy of Science. 25: 1-33.

1975.- McMullin, Ernan: "History and Philosophy of Science: A Marriage of Convenience?" en PSA 1974. pp.515-31. (vol.32 de los Boston Studies in the Philosophy of Science) Dordrecht. D. Reidel.

1976.- R.S. Cohen et al. (eds.): Essays in Memory of Imre Lakatos. Dordrecht. D.Reidel. (vol 39 de los Boston Studies in the Philosophy of Science).

1977.- Butts, R.E. y Hintikka, J. (eds.): Historical and Philosophical Dimensions of Logic, Methodology and Philosophy of Science (vol. 12 de los University of Western Ontario Series in Philosophy of Science). Dordrecht. D. Reidel.

1977.- Burian Richard, M.: "More than a Marriage of Convenience: On the Inextricability of History and Philosophy of Science", en Philosophy of Science, 44, $1-42$.

1978. - Radnitzky G. y Andersson G. (eds.): Progress and Rationality in Science (vol. 58 de los Boston Studies in the Philosophy of Science) Dordrecht. D. Reidel.

15 Véase, sobre todo, la primera parte del artículo de Kuhn en el Coloquio de Londres de 1965, que presidía Popper, en I.Lakatos y A. Musgrave (eds.) La crítica y el desarrollo del conocimiento, trad. de Francisco Hernán, Edit. Grijalbo, Barcelona 1975, pp. 81-111. 
propuesto por Kuhn es realmente adecuado para explicar el desarrollo científico?

Mi primera observación a este respecto es que paradigma, cualquiera que sea el significado que, además de los registrados ${ }^{16}$ por M. Masterman, Kuhn le diese dentro y fuera del texto de las Revoluciones, parece, a primera vista, una categoría o un metaconcepto, por así decirlo, que no pertenece por su naturaleza a la misma clase de categorías o conceptos que usamos para referirnos a las formulaciones directamente científicas tales como ley, hipótesis, experimento, comprobación, etc. Quizá, a primera vista, nos parezca semejante a una clase de conceptos meta-históricos capaces de expresar niveles diferentes de generalidad y de los cuales se sirve la historia para proporcionarnos sus explicaciones, o quizá a una clase de términos abstractos -idea, forma, causalidad,como suelen ser aquellos que usan los metafísicos. Pero, por otra parte, parece referirse demasiado ambiguamente a una entidad que actúa como si poseyese capacidad causal en algún sentido psicológico, epistémico o sociológico y, por tanto, historiable. Esta impresión aumenta cuando entre paradigma y práxis concreta de los científicos se da una correlación (¿causal?) tan fuerte como la que atribuye Kuhn a los episodios de ciencia normal -anómalos o no. De hecho Kuhn dedica un extenso análisis -las Segundas reflexiones- a este asunto. Lo que uno puede concluir de la lectura de este análisis es que Kuhn considera válida esta categoría conceptual para ser manejada desde dentro de la historia de la ciencia como categoría explicativa (causalmente o no) del acontecer científico. ${ }^{17}$ Además Kuhn, desde este punto de vista, concede a

16 En las "Segundas reflexiones acerca de los paradigmas" Kuhn no desdice a Masterman por haber catalogado hasta veintidós usos y sentidos del término en las La estructura de las reooluciones cientificas.

17 La noción más general es la de Visión-del-Mundo (World-View) pues, con 
esta categoría un mayor valor y superior en capacidad de determinación a otras, tales como teoría, modelo, programa, tradición, etc. Es superior en valor porque comprende mejor, esto es, refiere con mayor generalidad, tanto a los contenidos científicos de una disciplina como a los agentes histórico-causales que intervienen en ella. Esta doble referencia del término es, desde luego, una de las razones de su ambigüedad. Ya Ernan McMullin ${ }^{18}$ se hizo cargo del diferente alcance que tiene -o que venía teniendo en la literatura específica- el término "ciencia" pospuesto a Filosofía de... y pospuesto a Historia de... En el primer caso 'Ciencia' $\left(\mathrm{C}_{1}\right)$ es asumida como un producto final de la actividad de los científicos, en el segundo caso 'Ciencia' $\left(C_{2}\right)$ es interpretada como el proceso conducente a la producción de $\mathrm{C}_{1}$. McMullin reconoce que entre estos dos sentidos de "Ciencia" se da un continuo extenso con posibles valores intermedios en la historiografía de la ciencia, lo que se materializa en diferentes concepciones de la historia de la "Ciencia", cuyos extremos serían $\mathrm{HC}_{1}$ y $\mathrm{HC}_{2}$.

A su vez la "Filosofía" de la ciencia admite, para McMullin, tres acepciones diferentes: Filosofía de la ciencia desde la Metafísica (FCM), Filosofía de la ciencia desde la Lógica, (FCL) -ambás filosofias de la ciencia de carácter "externista" (FCE) respecto a la ciencia- mientras que desde un punto de vista "internista" cabe una Filosofía de la ciencia desde el análisis interno de lo que hacen los científicos (FCI) más que desde lo que "dicen" que hacen. A esta clase de FCI pertenecería, dentro de la taxonomía de McMullin, la Filosofía de la Ciencia incrustada en las Revoluciones de Kuhn,

muchos matices, Kuhn siempre ha mantenido que un científico que cambia de paradigma vive o trabaja en a different world. Con todo, a veces resulta identificado con alguna de sus funciones como ejemplar, matriz disciplinar, etc. En mi opinión paradigma menciona principalmente la estructura mental que condiciona la particular Visión-del-Mundo, pero no consiste en Aspecto-del-Mundo resultante.

18 E. McMullin: "The History and Philosophy of Science: A Taxonomy", en Roger H. Stuewer (ed.): Historical and Philosophical Perspectives of Science. Minnesota Studies in the Philosophy of Science, vol V. University of Minnesota Press. Minneapolis, 1970. pp. 12-67. 
Filosofía a la que se da apoyo -los paradigmas, p.e.- desde la Ciencia, tanto $\mathrm{C}_{1}$ como $\mathrm{C}_{2}$.

En este ensayo McMullin dedica un breve comentario a Kuhn; ${ }^{19}$ resumido en sus propias palabras, dice:

\begin{abstract}
"Su tesis fundamental es que los cambios de paradigma no pueden justificarse sobre bases empíricas ni incluso racionales, aunque post factum siempre pueda hacerse un esfuerzo para dotarlos de semejante racionalización. Es esta una tesis arriesgada; niega la posibilidad de cualquier clase de FCL aplicable a los avances significativos de la ciencia. Además, a muchos les ha parecido que pone en entredicho todo el conjunto de concepciones formalistas sobre las que se ha basado el análisis lógico de la ciencia. La Historio-Filosofía de la Ciencia (HFC) de Kuhn es así la antítesis de la FCL, lo que puede ayudar a explicar el calor que ha suscitado. Para nosotros, continúa McMullin, lo importante a propósito de esto es que únicamente la Historia de la Ciencia puede servir como evidencia en su apoyo. Es una tesis filosófica sobre la naturaleza de $C_{2}$ y sobre las características transformacionales de $C_{1}$. No puede derivarse de una teoría general del conocimiento, ni puede tampoco apoyarse en ụn análisis lógico formal".
\end{abstract}

El artículo que acabo de mencionar no añade mucha claridad (salvo la conclusión de que es una tesis filosófica sobre la naturaleza de $C_{2}$ y sobre las características transformacionales de $C_{1}$ ) a la cuestión con la que Herbert Feigl cree en su breve artículo inicial -Beyond Peaceful Coexistence- que deberian habérselas en ese momento: "...hay muchas fuentes y tipos de malentendidos entre historiadores de la ciencia y filósofos de la ciencia". ${ }^{20}$ Otros, en cambio, tratan de plantear la cuestión más crudamente. Wesley C. Salmon en su artículo "Bayes's Theorem and the History of Science" se plantea el problema en términos de la distinción de contextos -de descubrimiento y de justificación.

"Los críticos del punto de vista reconstruccionista, dice, han sugerido que éste deja el estudio de la ciencia vital, viviente y en desarrollo para los historiadores, relegando de paso la filosofía de la ciencia a la disección de cadáveres -no del

Loc. cit., pp. 61-62.

20 Steuwer R.H.: op. cit. p. 3. 
cuerpo de los científicos, sino de teorías que se han desarrollado hasta el punto del estancamiento y la osificación. Según tales críticos el estudio de la ciencia terminada no es el estudio de la ciencia en absoluto. Uno no puede comprender la ciencia salvo que vea cómo se desarrolla; comprender la estructura lógica de la ciencia es necesario para entender el cambio científico y la revolución científica. Algunos filósofos han sostenido, en consecuencia, que la filosofía de la ciencia debe ocuparse tanto de la lógica del descubrimiento como de la lógica de la justificación. La filosofía de la ciencia, se ha dicho, no puede seguir sin el estudio de la historia de la ciencia. Tales argumentos han llevado a una completa distinción entre descubrimiento y justificación. (Aquí en nota p.p.: E.g. Thomas S. Kuhn, The Structure of Scientific Reoolutions (Chicago: Univ. of Chicago Press, 1962), p. 9."21

En suma, una controversia que se centra en si la historia daria cuenta de los descubrimientos y, eventualmente, del desarrollo o progreso de la ciencia, mientras que la filosofía se centraría en diseccionar primero y reconstruir después la ciencia yacente en las bibliotecas según un patrón propio, lógico o metafísico según los casos. De dicha reconstrucción, además, surgiría una pretendida metodología prescriptiva con moraleja para los científicos: Si la ciencia no fue asi, asi debió ser y asi debería ser en el futuro. Los filósofos se mantienen en sus trece diciendo que, si la ciencia es un conocimiento, entonces pueden preguntar con todo derecho por la naturaleza, condiciones de validez y estructura de ese conocimiento.

Los historiadores, por su parte, tratarían de reconstruir la secuencia de hechos científicos como realmente ocurrieron. Ahora bien, no todos los hechos, sino sólo los que consideran positivos o relevantes para el progreso o, en sus términos, los que han tenido fallos incluidos- algo que ver con el éxito.

Si la ciencia es como debe ser, entonces podríamos conformarnos con descripciones lo más completas que se puedan dar de todos aquellos procesos tanto mentales como sociales y secuencias fácticas que han ido generando las ciencias que conocemos. Pero, en este caso, ¿cuál es el modo de saber cómo debe ser la ciencia?

21 Stuewer, op. cit., pp. 69-70. 
De no saber esto de algún modo no parece posible reconstruir la ciencia en términos de desarrollo y habriamos de limitarnos a reconstruirla en meros términos de proceso, a lo sumo darwinista, de antes y después. La reconstrucción histórica resuelve el problema drásticamente (¿dogmáticamente?), diciendo: la ciencia, en tanto que proceso histórico, ${ }^{22}$ está naturalmente dado $\mathrm{y}$, por tanto, no hay ideal alguno al que debiera ajustarse, como tampoco los demás procesos naturales obedecen a ningún ideal. No parece que haya una cuestión tal como la del deber ser o la de la metodología prescriptiva surgida de los laboratorios reconstructivistas.

La segunda observación viene sugerida por la propia noción de Historia que aparece en la obra y en las controversias a propósito de ella. Primero, la idea de Historia como "narración de eventos" $\left(\mathrm{H}_{1}\right)$ (en este caso científicos) entraña sus propias exigencias (veracidad, temporalidad, incompletud, p.e.) de cuyo cumplimiento se ocupa, implícita o explícitamente, la metodología de la Historia mediante algunos requisitos que los historiadores de la ciencia, al igual que los demás, han tratado de observar, según parece, casi siempre. El resultado, grosso modo, es el establecimiento de algunos eventos con la máxima seguridad de que "tal es el caso", la fijación temporal de dichos eventos con respecto a otros y la descripción de algunas de las circunstancias concomitantes que pueden "contrastarse" de acuerdo con los métodos históricos de contrastación -archivos, manuscritos, correspondencia directa e indirecta, etc. Simplificando mucho, podemos decir que

22 Feyerabend objetó al manuscrito de las Revoluciones en este sentido: This manner of presentation you share with Hegel and with Wittgenstein; and with all those who say, when engaging in a political enterprise, that "history will be our judge". Cfr. Paul Hoyningen-Huene: "Two Letters of Paul Feyerabend to Thomas S. Ruhn on a Draft of The Structure of Scientific Revolutions" en Studies in History and Philosophy of Science, 26-3 (1995), p. 355. 
esta clase de Historia consiste en la yuxtaposición de hechos singulares, esta guerra, aquella revolución política, aquel tratado de paz, etc.

Pero, en segundo lugar, la Historia, desde Tucídides, se extiende a la "narración causal de eventos" $\left(\mathrm{H}_{2}\right)$. Se trata de la fórmula "tal y tal evento ocurrieron por esto y por aquello". Es obvio que esta fórmula entraña requisitos adicionales respecto a los que puedan satisfacer a una "buena" $\mathrm{H}_{1}$. La introducción de la relación "por" entraña una dificultad adicional en el caso de los eventos históricos típicos: no es difícil establecer que "Cesar murió porque Bruto le asestó tres puñaladas", aunque es más difícil establecer que "Bruto apuñaló tres veces a Cesar porque odiaba su dictadura". En este segundo caso la relación "causal" es sólo comprensible, mientras que en el primero podría resultar, desde el punto de vista empírico, mucho menos problemática. Explicar un evento -la muerte de Cesar- por otro evento -las puñaladas de Bruto- es el tipo de explicación histórica que reproduce mejor el modelo de explicación causal frecuente en las ciencias naturales. El otro tipo de explicación, la de un evento que es una acción humana -las puñaladas de Bruto- mediante la introducción de un motivo -odio a la dictadura- no pasa de ser una problemática analogía respecto a la primera.

Ahora bien, al leer las Revoluciones nos encontramos múltiples argumentos, aunque formulados difusa y retóricamente, que se ajustan al formato del motivo que explica una acción. Trataré de ilustrar esto con un ejemplo:

"El hombre que adopta un nuevo paradigma en una de sus primeras etapas, con frecuencia deberá hacerlo, a pesar de las pruebas, por la resolución de los problemas. $O$ sea, deberá tener fe en que el nuevo paradigma tendrá éxito al enfrentarse a los muchos problemas que se presenten en su camino, sabiendo sólo que el paradigma antiguo ha fallado en algunos casos. Una decisión de esta índole sólo puede tomarse con base en la $\mathrm{fe}^{\mathrm{\prime 23}}$.

${ }^{23}$ La Estructura de las Revoluciones Cientificas. Trad. de Agustín Contín. México: F.C.E., 1971. p. 244. 
Pese a la oscuridad añadida por el traductor, este texto -por lo demás característico del estilo de Kuhn- aparentemente pretende convencernos de que un científico adopta un nuevo paradigma porque tiene fe en determinadas propiedades del mismo. Desde el punto de vista histórico podríamos admitir como hechos i) que el científico en cuestión tenía en su mente, en un momento dado, un paradigma antiguo, de cuya presencia la historia de la ciencia puede tener indicios racionales de muy diferentes modos; ii) que en un momento posterior ese científico tiene en su mente un nuevo paradigma, a juzgar por los nuevos indicios o, si se quiere, hasta evidencias. Desde luego, la afirmación conjunta de estos dos hechos por parte de un historiador de la ciencia entraña la afirmación implícita de que dicho científico ha cambiado de paradigma. Pero esto, claramente, no es una explicación. Es sólo $\mathrm{H}_{1}$. Vendría a ser $\mathrm{H}_{2}$ únicamente cuando i) y ii) se hallen conectados explicativamente, esto es, cuando el porque fuese explicativamente relevante. $Y$ para que lo fuese habría que demostrar, al menos, que es condición necesaria para la ocurrencia del explanandum. En mi opinión Kuhn no hace esto en ninguno de los casos en que utiliza este modelo argumental. ${ }^{24}$ En todos esos casos las alternativas posibles

24 Ahora invito al lector a releer con la mente puesta en este problema el artículo de Kuhn "Las relaciones entre la historia y la filosofía de la ciencia" en La Tensión Esencial, donde trata de explicar cómo la historia de la ciencia es una posible fuente para una reconstrucción racional de la ciencia (p.39) obviamente de naturaleza explicativa, aunque sin leyes implícitas o "encubiertas" como dice el traductor. La carga explicativa reside, ahora, en una especie de "reconocimiento de semejanzas" entre lo "explicado" y algo previo conocido. "El niño ha visto antes fotografías semejantes a ésta, mientras que el historiador ha contemplado similares pautas de conducta. Creo que ese reconocimiento de la similitud es previo a cualquier respuesta sobre la clase de similitud lograda... Es global, no reductible a un conjunto único de criterios previos más elementales que la propia relación de similitud... Si la historia es explicativa, ello no se debe a que sus narraciones estén apoyadas por leyes generales. Se debe más bien a que el lector dice: 'A hora ya sé lo que ocurrió', mientras simultáneamente afirma 'Ahora esto tiene sentido; ahora entiendo; lo que antes fue para mí una mera lista de hechos ahora se ha reconocido en una pauta reconocible'". Esta percepción de "la realidad histórica" como revivencia del pasado permite que "Cuando el historiador de la ciencia surge de la contemplación de las fuentes y de la construcción de una narración, puede tener entonces el derecho a proclamar que está familiarizado con los puntos esenciales: si dice luego No puedo construir 
son muchas e igualmente explicativas. Valdría igual, p.e. decir: un científico adopta un nuevo paradigma porque no tiene fe en el antiguo. Pero también, porque siente curiosidad por alguna idea nueva o porque le gusta divertirse haciendo experimentos mentales o porque quiere fastidiar a algün colega, etc.

Pero si los hechos del tipo $\mathrm{H}_{1}$ que hemos esquematizado como i) y ii) se utilizan como "confirmaciones" de la explicación por la fe en el nuevo paradigma, es evidente que servirían también para los demás ejemplos.

Tampoco deberíamos pasar por alto los matices metodológicos indirectamente introducidos en la frase deberá tener fe en que el nuevo paradigma tendrá éxito al enfrentarse a los muchos problemas que se presenten en su camino. No es neutral la expresión deberá tener fe, pues insinúa, aunque no tenga formato prescriptivo, o un mandato o un requisito necesario. Tras toda esta forma de argumentación se oculta un doble lenguaje, referido por un lado a hechos y por otro a reglas metodológicas: así en la frase que vengo comentando nos encontramos con que la fe en el nuevo paradigma se centra en creer que tendrá éxito al enfrentarse a los muchos problemas... donde se está dando por supuesto que hay formas buenas -las exitosas- de resolver problemas y por consiguiente, que debe haber algún criterio para decidir cuales son buenas o malas o mejores o peores. Pero ¿de qué naturaleza es este criterio? No parece que una solución sea buena por el mero hecho de depender del nuevo paradigma, ni mala por depender del viejo.

una narración viable sin concederle un lugar central a esos aspectos de la ciencia. que los filósofos pasan por alto, como tampoco puedo hallar huellas de elementos que ellos consideran esenciales', entonces merece que se le oiga. Lo que está diciendo es que la empresa reconstruida por el filósofo no es la ciencia, en cuanto a algunos de sus puntos esenciales". 
Todavía me parece necesario llamar la atención sobre otra grave ambigüedad en las Revoluciones y más concretamente en el planteamiento de Kuhn: Las revoluciones, i.e. su naturaleza y su dinámica. Una observación inicial es que Revolución es un nombre más adecuado para un proceso -no instantáneo ni siquiera generalmente breve llevado a cabo por muchos y no necesariamente confabulados protagonistas. La dispersión y la no coincidencia de programas de investigación entre los creadores de una nueva teoría (tomo aquí teoría como elemento sintomático mínimo en un nuevo paradigma) me inducen a creer que un paradigma no surge nítido y acabado de la mente de un científico, sino que acaba siendo una formulación de acuerdos mínimos entre científicos que investigan diferentes problemas, sean estos anomalías o no respecto a otro paradigma. Esto quiere decir que los paradigmas, en tanto que "conjuntos compartidos de..." no funcionan tanto prospectivamente como, sobre todo, retrospectivamente. $Y$ esto me lleva a sospechar que un nuevo paradigma tiene más de post que de pre-revolucionario -más resultado que causa de cambio. Lo que quiero decir es que los acuerdos mínimos post-revolucionarios entre científicos constituirian un nuevo paradigma, siempre que la revolución fuese algo más que una tormenta en un vaso de agua. También se podría decir que a un período de ciencia normal, sin apenas anomalías en su desarrollo cotidiano, podríamos describirlo como revolucionario si un científico realizando tareas de ciencia normal Herzt, Roentgen, Mme. Curie-se topa de repente con un fenómeno no descrito aún o no predicho por la ciencia que practica. En mi opinión Revolución es un término adecuado para referirse retrospectivamente a un proceso que acaba en la cristalización de un nuevo paradigma $y$, por consiguiente, las revoluciones jamás han sido ni programas científicos de investigación ni menos resultado de nuevos paradigmas. La caracterización que hace Kuhn de la ciencia normal seguramente es aceptable cuando se trata de ciencia "escolar" o para pre-graduados -concepto válido como instrumento 
didáctico y aproximativo. Pero en los laboratorios, en los departamentos o en los institutos, donde trabajan -no en régimen de esclavos o sirvientes del Gran Jefe- personas en competencia continua, existen menos acuerdos que desacuerdos entre los miembros de esos equipos, aunque en los años 40-60 la competen$\mathrm{Cia}^{25}$ fuese menor. En la actualidad, y dadas las enormes poblaciones de científicos en ejercicio, su rivalidad les lleva a explorar, a formular $y$, consiguientemente, a ensayar continuamente alternativas a lo que cada uno sabe que está haciendo su vecino. Este estado de cosas tiene poco, si es que algo, de ciencia normal y más bien resulta en sí mismo una especie de revolución permanente, al menos en los centros más avanzados. Si esto es así sobrarian las crisis y los nuevos paradigmas para que se produjesen cada día pequeñas revoluciones. ${ }^{26}$ Bastaría, pues, con ellas, es decir, con el juego ordinario de sus contrastes para acabar constituyendo un consenso de minimos.

25 Aquí valdría la pena estudiar la estructura de la ciencia norteamericana de esos años con la que Kuhn estaba familiarizado. Los programas científicos, entonces, se hallaban fuertemente cohesionados por los encargos del Gobierno, de las grandes corporaciones, etc. La acepción de paradigma que en su Postscriptum de 1969 denomina Kuhn sociológico, parece una descripción de aquellos equipos. "La completa constelación de creencias, valores, técnicas, y así sucesivamente, compartidas por los miembros de una comunidad dada" (p. 269 de la ed. española) debió ocurrir justamente en aquellos medios y momentos.

${ }_{26}$ Quizá estoy interpretando a Kuhn mismo de manera más radical de lo que a él le gustaria; que el sabio lector juzgue: "But in Structure the gestalt switch is repeatedly used also as a model for what happens to a group, and that use now seems to me mistaken. Goups do not have experiences except insofar as all their members do. And there are no experiences, gestalt switches or other, that all the members of a scientific community must share in the course of a revolution. Reoolutions should be described not in terms of group experience but in terms of the varied experiences of individual group members. Indeed, that onriety itself turns out to play an essential role in the eoolution of scientific knowledge" (las cursivas son mias). Asi lo decía Kuhn en el "Foreword" a Reconstructing Scientific Reoolutions de P. Hoyningen-Huene en Agosto de 1988. 
Poco a poco estoy proponiendo que paradigmas, revoluciones, crisis y ciencia normal son términos -al igual que las relaciones que Kuhn establece entre ellos- no pertenecientes directamente a la Historia de la ciencia que he etiquetado como $\mathrm{H}_{1}$, aunque no negaré que cumplen algún papel en el nivel etiquetado como $\mathrm{H}_{2}$. Pero esta última clase de Historia -en tanto que es explicación o, por lo menos, comprensión de $\mathrm{H}_{1}$, depende de su propia teoría o cosmovisión o ideología o lo que se quiera. En una crítica enormemente perspicaz que hizo Feyerabend ${ }^{27}$ al borrador de las Revoluciones le dice literalmente "What you are writing is not just history. It is ideology covered up as history" ${ }^{128}$ y lo repite no más suavemente en su contribución -Consuelos para el especialista- al Coloquio de Londres. Feyerabend le pide a Kuhn que distinga entre hechos, interpretaciones y punto de vista desde el cual se hacen estas últimas porque piensa que "es posible escribir historia de tal modo que el lector siempre sea consciente de la ideología o punto de vista de uno a la vez que (siempre sea consciente) de la posibilidad de una interpretación alternativa de los hechos históricos". ${ }^{29}$

La pregunta ahora, según esto, debería ser por el punto de vista desde el que Kuhn interpretó los hechos históricos. Siguiendo, por un momento todavía, a Feyerabend deberíamos saber primero si dicho punto de vista era un punto de vista filosófico o más bien ideológico o algo de ambos.

En su ensayo Las relaciones entre la historia y la filosofía de la ciencia $^{30}$, escrito en 1968 y revisado para su publicación alemana en 1976, deja claro que a la sazón consideraba irreductibles estas

27 Cfr. Paul Hoyningen-Huene: "Two Letters of Paul Feyerabend to Thomas S. Kuhn on a Draft of Structure of Scientific Revolutions", en Studies in History and Philosophy of Science, vol. 26-3 (1995), pp. 353-387.

28 Ibid., p. 355.

29 Ibid. (Las cursivas son de Feyerabend.)

${ }^{30}$ Cfr. La tensión Esencial. Madrid-México: F.C.E., 1982. pp. 27-45. 
dos formas de contemplar la ciencia, pese a que se podrían practicar alternadamente. Debería haber un diálogo entre ellas, pero "tal diálogo debe ser interdisciplinario y no intradisciplinario". ${ }^{31}$ Esto es así porque "la narración histórica debe hacer plausibles y comprensibles los acontecimientos que describe. En cierto sentido... la historia es una empresa explicatoria... El filósofo, por otra parte, trata ante todo de llegar a generalizaciones explícitas y especialmente a las que poseen validez universal". ${ }^{32}$ Afirma que, sin embargo, "pueden ser practicadas alternadamente, trabajando de tiempo en tiempo en problemas históricos y de cuando en cuando sobre problemas filosóficos. Como es obvio que mi manera de trabajar es esta última, creo firmemente que tal cosa puede hacerse". 33

Además creía Kuhn que, puesto que "las filosofías de la ciencia y del derecho se singularizan porque se dirigen a campos acerca de los cuales el filósofo como tal sabe muy poco", ${ }^{34}$ estos filósofos ignorantes de la ciencia sobre la cual hacen filosofía deberían aprender algo de ella en las aulas de los historiadores. Y así aprenderían a descubrir que "para el historiador, o por lo menos para mí, las teorías, en ciertos aspectos esenciales, son holísticas. Es decir, en la medida en que el historiador pueda decir que siempre han existido -aunque no siempre en las formas que cómodamente pueden describirse como científicas. Acerca de estos procesos sabemos muy poco, y nuestro conocimiento no avanzará más mientras no aprendamos a reconstruir teorías seleccionadas del pasado. Hoy en día, los que enseñan a hacer este trabajo son historiadores, no filósofos". 35

A juzgar por el ejemplo que propone Kuhn para, desde él, llegar a la conclusión que acabo de citar, aprender a reconstruir es

\footnotetext{
Loc. cit., p. 28.

Loc. cit., p. 29.

Ibid.

Loc. cit., p. 37.

Loc. cit., p. 45.
} 
aprender, p.e., a interpretar en su contexto histórico conceptual y cultural la Física de Aristóteles, que es aquí su ejemplo. El proceso de lectura y acomodación conceptual necesario para llegar a entender bien la teoría aristotélica del cambio cualitativo bajo el formato de la cuádruple causalidad sería p.e. un modelo a seguir en todos los textos que haya de leer un historiador. Tras ello su narración resultaria adecuada y llevaria al lector a comprender lo que "realmente pasó" y por ello dicha narración sería explicativa, es decir, habría dado cuenta completa de la ciencia, sin residuo posible fuera del devenir histórico evidenciado en la narración. Pero, como argumentaba Feyerabend en la carta mencionada más arriba, "nadie pensará que la historia del crimen justifica el crimen, ni que muestra que el crimen tiene una 'razón' inherente o una inherente moralidad en sí mismo".

La idea de que la realidad de las cosas se comprende mediante una especie de revivencia de su formato histórico completo pudiera tener algún interés, si el acento en el término completo fuese muy fuerte. El conjunto de items y relaciones exigido por esta idea de completud es, desde un punto de vista extensional, inabarcable para cualquier forma de narración y Kuhn matiza esto con su expresión puntos esenciales que serían el objeto de la narración. Pero, ¿cómo saber que son esenciales? El historiador sí lo sabe, pues, "no es mero accidente que, desde antes que comience a escribir, sepa el final de su narración lo mismo que el comienzo. No puede escribirse la historia sin esa información". ${ }^{36}$

\section{V}

La pregunta que nos haciamos al principio del epigrafe anterior parece ahora admitir otra formulación: ¿Qué claves han de guiar al historiador (Kuhn) cuando lee textos cientificos de cualquier época? Esto

${ }^{36}$ Loc. cit., p. 41. 
equivale a preguntarse por los esquemas previos -los aprioriideológicos, filosóficos o lo que sean.

Una investigación histórica profunda de una especialidad dada, en un momento dado, revela un conjunto de ilustraciones recurrentes y casi normalizadas de diversas teorías en sus aplicaciones conceptuales, instrumentales y de observación. Esos son los paradigmas de la comunidad revelados en sus libros de texto, sus conferencias y sus ejercicios de laboratorio.... A pesar de las ambigüedades ocasionales, los paradigmas de una comunidad científica madura pueden determinarse con relativa facilidad. ${ }^{37}$

Independientemente del alcance que pueda tener el término, lo que buscará un historiador kuhniano en un texto o en una colección documental será, antes que nada, el paradigma a que obedecen esos textos investigados. Es obvio que esta búsqueda presupone i) la existencia de paradigma y ii) la accesibilidad al mismo por parte del historiador $y$, finalmente, también es obvio que aquí se presupone iii) que el historiador dispondrá de alguna clave especial para poder aislar, reconocer e interpretar los diferentes paradigmas de las diferentes escuelas o comunidades $y$, además, dispondrá de un lenguaje adecuado (¿neutral?) para explicar en términos del mismo la sucesiva secuencia de paradigmas que constituyen la estructura del desarrollo de la ciencia; pongamos que es el lenguaje o discurso histórico.

No dejaré ahora de proponer al lector una pregunta inquietante que surge aquí, y en otros contextos kuhnianos: ¿Paradigma es una entidad -ideal, por supuesto- perteneciente a la ciencia o perteneciente al historiador de la ciencia? Esto es, ¿Los científicos usan paradigmas o usan teorias, leyes, hipótesis, experimentos y cosas así? Y más radicalmente ¿Los 'paradigmas' pertenecen a la ontología de la ciencia? Para mí la respuesta es No. ${ }^{38}$ Los paradigmas, las revoluciones, la ciencia normal y todas esas cosas, son

37 Reooluciones.., p. 80.

36 De igual modo que los diferenciales o los infinitésimos no pertenecen a la ontologia del tiempo o del espacio, ni en general a la ontología de la Física, como he discutido tantas veces con mi querido amigo el Dr. Selles. 
esquemas o puntos de vista o categorías o instrumental teórico del historiador, mediante el cual trata de comprender o explicar o revivir la ciencia que investiga en los textos de diferente clase. Esto nos llevaría inmediatamente a formulaciones aparentemente (si no es que también realmente) anti-kuhnianas: La ciencia no es en sí misma ni revolucionaria ni normal, ni sufre crisis ni sale de ellas; simplemente es lo que hacen los científicos.

Los historiadores harán bien en presentarnos lo que hacen los científicos de diferentes formas, acumulativas, revolucionarias, recurrentes, evolutivas, en flujo o estancadas y cuantas otras se les ocurran. $Y$, desde luego, hay que reconocerles la libertad de elegir su propio instrumental teórico cuando esa presentación, sobre todo, quiere ser también interpretativa o explicativa. Y a la vez ellos deberían reconocer que su elección entraña, al menos, alguna consecuencia para la historia que van a reconstruir. Sólo que, por afortunada que resulte la reconstrucción histórica, será siempre una reconstrucción History-laden y, en su presentación, debería tener presente aquella objeción que itan tempranamente! hiciera Feyerabend al manuscrito de las Revoluciones:

..."you use a kind of double-talk where every assertion may be read in two ways, as the report of a historical fact, and as a methodological rule. You thereby take your readers in. You present your material in such a manner that (at least for the period following the introduction of paradigms) history seems to satisfy the principle that ALLES WIRKLICHE IST VERNÜNFTIG so that evaluations can then be derived from historical study. It is this way of presentation which I find objectionable. I do not object to your belief that once a paradigm has been found a scientist should not waste his time looking for alternatives but try working it out. That is, I do objet to this belief, and I shall have very soon to say a little more about it. What I do object to most emphatically is the way you present this belief of yours; you present it no as a demand, but as something that is an obvious consequence of historical facts. Or rather, you do not even talk about this belief, you let it as it were emerge from history as if history could tell you anything about the way you should run science (is does not imply ought!). It is this bewitching way of presentation to 
which I object most, the fact that you take your readers in rather than trying to persuade them". 39

\section{VI}

Quizá era esa creencia callada -de la que nunca hablaba, pero lo haria más tarde- lo que a Feyerabend le parecía ideología. En mi opinión podría denominarse epistemología o filosofía o, acaso, metafísica. No hago cuestión del nombre. Lo que quiero decir, y trataré de poner en claro, es que Kuhn abordó la historia de la ciencia desde una concepción filosófica, quizá poco explícita y quizá también algo sincréticamente elaborada, pero que condicionó buena parte de su visión de la ciencia. Esta concepción filosófica determinante, para decirlo de manera drástica, es su teoría del conocimiento.

Kuhn se refiere elípticamente a su filosofía o a sus trabajos filosóficos en múltiples ocasiones, en las Revoluciones, primero, en La Tensión Esencial, y en muchos artículos, sobre todo desde $1983,{ }^{40}$ culminando, hasta donde yo conozco, en su contribución "Afterwords" a World Changes, si bien anuncia aquí un libro que traía entre manos por aquellos dias de Julio de 1990 y cuyo embrión venía dado por los manuscritos utilizados para sus tres Conferencias Shearman del University College de Londres en 1987. Honestamente confiesa Kuhn que en los días en que escribía las Revoluciones su familiaridad con la Filosofía de la Ciencia $-y$

39 "Two letters of Paul Feyerabend...", loc. cit., p. 355 (primera carta).

40 P. e. "Commensurability, Comparability, Communicability" en PSA 1982. P.D. Asquith y T. Nickles (eds.) Proceedings of the 1982 Biennial Meeting of the Philosophy of Science Association. East Lansing, 1983, pp. 669-688; y "Response to Commentaries" pp. 712-716. "Rationality and Theory Choice" en Journal of Philosophy (1983) n' 80, pp. 563-570. "Possible Worlds in History of Science", en S. Allén (ed.), Possible Worlds in Humanities, Arts, and Sciences. de Gruyter, Berlín, 1989, pp. 9-32. "Dubbing and Redubbing: the Vulnerability of Rigid Designation", en C.W. Savage (ed.): Scientific Theories. Minnesota Studies in Phlosophy of Science 14. Univ. of Minnesota Press, Minneapolis. 1990, pp. 298-318. etc. 
probablemente con la Filosofía en general- no era demasiado profunda. ${ }^{41}$ Además insinúa que, al verse obligado a revisar los términos en que presentó su reconstrucción del desarrollo científico, se encontró con problemas filosóficos que no había sospechado. Sin ir más lejos, en el 'Prefacio' a La Tensión Esencial, se hace eco del problema que ha introducido en su concepción al mantener que el conocimiento cientifico es intrinsecamente un producto de grupo y que es imposible entender tanto su eficacia peculiar como la forma de su desarrollo sin hacer referencia a la naturaleza especial de los grupos que la producen, ${ }^{42}$ tesis que abrió la entrada a los sociólogos de la ciencia con su inevitable relativización de valores $e$ intereses. Mucho más significativo resultó el problema de la posesión de un lenguaje común o dialecto especial ${ }^{43}$ como elemento que liga a los miembros de una comunidad científica y los diferencia (cursiva mía) de los miembros de otra aparentemente igual'. Los problemas de inconmensurabilidad, traducción y cambio de significado no dejan ya en paz a Kuhn y le arrastran a una discusión netamente filosófica. Según creo, es esta discusión la que va llevando a Kuhn hacia una filosofía más refinada, explícita $y$, eventualmente, no siempre compatible con sus más crudos puntos de vista iniciales.

En el ya citado estudio de Hoyningen-Huene se evidencia esta progresiva deriva filosófica p.e. a propósito de lo que Hoyningen denomina Phenomenal World (mundo de objetos conocidos científicamente) que en Kuhn aparecía de varios modos, pero sobre todo como World-View o similares. Desde 1969 en adelante se producen revisiones que afectan al primitivo esquema conceptual de manera esencial. Sucintamente expuesto sería como sigue.

En el esquema inicial se diseñaba un molde rígido para toda ciencia madura, molde que Kuhn denominó estructura. Los

41 Whatever role the problems encountred by positivism may have played in the background for The Stucture of Scientific Revolutions, my knowledge of the literature that attempted to deal with those problems was decidedly sketchy when the book was written. En P. Horwich (ed.) : World Changes. "Afterwords", p. 313.

12 Loc. cit., p. 21.

4 Loc. cit., p. 22. 
materiales de este artefacto eran las comunidades de científicos y los textos o fuentes históricas dejados por los mismos. El elemento formal unificador de estos materiales sería el paradigma o 'visióndel-mundo' que compartían. Esta 'visión' tenía propiedades deterministas sobre la actividad de los científicos. Determinaba lo que podían hacer y también lo que no podían hacer, entre esto estaba la imposibilidad de ver el mundo de otro modo. Con todo, algunas veces dentro de la comunidad y de sus prácticas científicas aparecían (graves) anomalías -seres literalmente de otro mundo. El artefacto podría responder de dos maneras: i) Puzzle-solving, mediante refinamientos adecuados de los elementos conformadores de la 'visión-del-mundo' en que se habita; ii) World-change, cambiando de visión-del-mundo. Pero esto entraña, además de Revolución, cambio de Paradigma y en suma cambio o acomodación del artefacto, pasar de un lenguaje a otro. Aunque Kuhn intuyó que esto comportaba perturbaciones graves para los miembros de la comunidad, le pareció al principio que de manera disciplinada se convertirian a la nueva 'visión-del-mundo'. Aunque la conversión ocurriese mediante un acto de fe, ello no evitaba un nuevo proceso de aprendizaje de nuevos conceptos empíricos, de nuevas técnicas de trabajo, de nuevas teorías y así sucesivamente pues, después de una revolución los científicos trabajan en un mundo diferen$t e e^{44}$ Tres veces más en este famoso Cap. $X$ dice Kuhn esto mismo y algunas cosas más con otras palabras. Muchos estudiosos ${ }^{45}$ de su pensamiento se han esforzado en aclarar cómo se puede entender que con el cambio de un paradigma a otro los científicos vivan en, trabajen en, vean, contemplen... etc. otro mundo' y a la vez 'el mundo externo, el mundo del que proceden los estímulos

4 Revoluciones, p. 211.

45 El libro colectivo editado por Paul Horwich: World Changes, ya citado, permite hacerse una idea de lo que ha sido este debate y en qué ha venido a parar, junto con el punto al que llegó el propio Kuhn hacia mediados de 1990. Me seguire refiriendo a estos textos en lo que sigue. Pero, además, este problema lleva directamente al de la inconmensurabilidad que ha hecho correr ríos de tinta. Puede verse el repertorio que recoge Hoyningen-Huene en su Reconstructing Scientific Reoolutions, en p. 207 n. 58. 
que recibe el científico, el mundo de la vida ordinaria que continúa como antes... etc.' no cambie. Resulta inmediatamente obvio que esta tesis no se inscribe fácilmente entre las posiciones realistas. Adquirir un paradigma parece tan perturbador como adquirir una cámara fotográfica con objetivo deformante y cambiar de objetivo (cambiar de paradigma) sólo significaría sustituir unas deformaciones por otras. Además la sustitución añade problemas adicionales, pues la nueva fotografía no sería superponible con la antigua, dado que no disponemos de ningún medio neutral que permita interpretar en cuál de las dos y cómo acontecen las deformidades. Son inconmensurables. Kuhn reconoce que este problema es el más importante de los que afectan a su proyecto: I begin with some anticipatory remarks on the topic that dominates my project: incommensurability and the nature of the conceptual divide between the developmental stages separated by what I once called "scientific revolutions". ${ }^{46}$ Antes y después de las Revoluciones sus esfuerzos por aclararse con esta cuestión central were extremely crude. ${ }^{47} Y$ añade, para desconsuelo de creyentes: Efforts to understand and refine it have been my primary and increasingly obsessive concern for thirty years, during the last five of which I've made what I take to be a rapid series of significant breakthroughs. ${ }^{48}$ Parece que el eco de esos fantasmas acompañó a Kuhn durante largos treinta años y que a partir de 1987 -yo ahora desconozco el final de la historia- intentó deshacerse de él mediante un breakthrough en su teoría del conocimiento. Nos asegura que "una excelente descripción de los primeros pasos de estos intentos aparece en el libro de Paul Hoyningen-Huene", y nos pone en la pista de sus pensamientos.

Puesto que el cambio de lenguaje -que en definitiva acompaña al cambio de paradigma- es cognitivamente significativo (pero no necesariamentedestructivo de la base empírica del lenguaje anterior) Kuhn se decide a investigar cómo cambian los conceptos

46 En "Afterwords". P. Horwich (ed.), World Changes, p. 314.

47 Loc. cit., p.315.

48 Loc. cit., p. 315. 
relevantes -generando con ello un cambio de World-view. Para ello quizá el primer paso debería consistir en relegar la inconmensurabilidad al mundo de los significados y mantener entre dos lenguajes la compatibilidad de usos. Basta con reconocer estructuras lingüisticas vinculadas al aprendizaje, presuntamente de carácter neuronal. Se trataria de kind-concepts cuyas funciones léxicas y taxonómicas admiten cambios sin rupturas drásticas. Dicho con sus palabras: what is required is a characteristic of kind and kind-terms in general. In the book I will suggest that this characteristic can be traced to, and on from, the evolution of neural mechanisms for reidentifying what Aristotle called "substances": things that, between their origin and demise, trace a lifeline through space over time. What emerges is a mental module that permits us to learn to recognize not only kinds of physical objet (e.g. elements, fields, and forces), but also of furniture, of government, of personality, and so on. ${ }^{49}$

Kuhn decía, hace mucho tiempo, que lo que decían los filósofos de la ciencia era absolutamente irreconocible por los científicos en ejercicio. No sabría yo decir cuánto de familiar será en los laboratorios la versión final del problema de Kuhn, pues I do talk of change in concepts and their names, in conceptual vocabulary, and in the structured conceptual lexicon that contains both kind-concepts and their names. Pero por irreconocible que resultase esto en los laboratorios, tampoco por ello habría razón alguna suficiente para negar a Kuhn la gloria de ser filósofo.

4 Loc. cit., p. 315. 